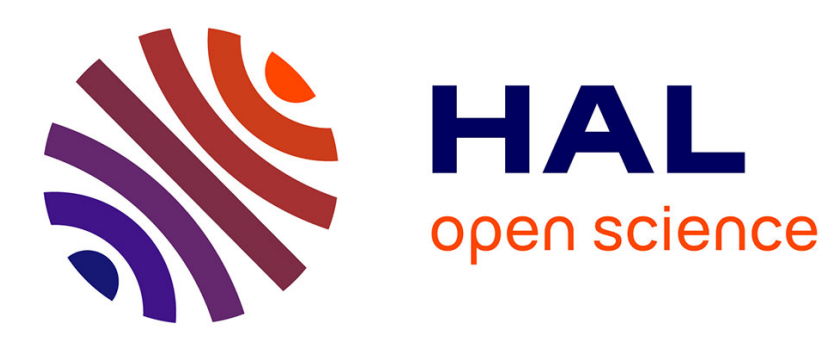

\title{
Lie symmetries of geodesic equations and projective collineations
}

\author{
Michael Tsamparlis, Andronikos Paliathanasis
}

\section{To cite this version:}

Michael Tsamparlis, Andronikos Paliathanasis. Lie symmetries of geodesic equations and projective collineations. Nonlinear Dynamics, 2010, 62 (1-2), pp.203-214. 10.1007/s11071-010-9710-x . hal00586134

\section{HAL Id: hal-00586134 \\ https://hal.science/hal-00586134}

Submitted on 15 Apr 2011

HAL is a multi-disciplinary open access archive for the deposit and dissemination of scientific research documents, whether they are published or not. The documents may come from teaching and research institutions in France or abroad, or from public or private research centers.
L'archive ouverte pluridisciplinaire HAL, est destinée au dépôt et à la diffusion de documents scientifiques de niveau recherche, publiés ou non, émanant des établissements d'enseignement et de recherche français ou étrangers, des laboratoires publics ou privés. 


\title{
Lie symmetries of geodesic equations and projective collineations
}

\author{
Michael Tsamparlis* and Andronikos Paliathanasis ${ }^{\dagger}$ \\ Department of Physics, Section of Astronomy-Astrophysics-Mechanics, \\ University of Athens, Panepistemiopolis, Athens 157 83, GREECE
}

\begin{abstract}
We prove a Theorem which relates the Lie symmetries of the geodesic equations in a Riemannian space with the collineations of the metric. We apply the results to Einstein spaces and spaces of constant curvature. Finally with examples we show the use of the results.
\end{abstract}

Keywords:

Geodesics, General Relativity Theory, Classical Mechanics, Collineations, Riemannian Space, Autoparallels, Lie Symmetries, Projective Collineations.

PACS - numbers: 2.40.Hw, 4.20.-q, 4.20.Jb, 04.20.Me, 03.20.+i, 02.40.Ky

\section{Motivation}

In a Riemannian space the affinely parameterized geodesics are determined uniquely by the metric. Therefore one should expect a close relation between the geodesics as a set of homogeneous ordinary differential equations (ODE) linear in the highest order term and quadratic non-linear in first order terms, and the metric as a second order symmetric tensor. A system of such ODEs is characterized (perhaps not fully) by its Lie symmetries and a metric by its collineations. Therefore it is reasonable to expect that the Lie symmetries of the system of geodesics of a metric will be closely related with the collineations of the metric. That such a relation exists it is easy to see by the following simple example. Consider on the Euclidian plane a family of straight lines parallel to the $x$-axis. These curves can be considered either as the integral curves of the ODE $\frac{d^{2} y}{d x^{2}}=0$ or as the geodesics of the Euclidian metric $d x^{2}+d y^{2}$. Subsequently consider a symmetry operation defined by a reshuffling of these lines without preserving necessarily their parametrization. According to the first interpretation this symmetry operation is a Lie symmetry of the ODE $\ddot{y}=0$ and according to the second interpretation it is a (special) projective symmetry of the Euclidian two dimensional metric.

What has been said for a Riemannian space can be generalized to a space in which there is only a linear connection. In this case the geodesics are called autoparallels (or paths) and they comprise again a system of ODEs linear in the highest order term and quadratic non-linear in the first order terms. In this case one is looking for relations between the Lie symmetries of the autoparallels and the projective or affine collineations of the connection.

The above matters have been discussed extensively in a series of interesting papers by Aminova [5], [2], [3], [4] who has given an answer. Furthermore in a recent work [6] they have considered the KVs of the metric and their relation to the Lie symmetries of the system of affinely parameterized geodesics of maximally symmetric spaces of low dimension. In the same paper a conjecture is made, which essentially says that the maximally symmetric spaces of non-vanishing curvature do not admit further Lie symmetries.

In the present paper we consider the set of autoparallels - not necessarily affinely parameterized - of a symmetric connection, and in section 4 we determine the conditions for Lie symmetries in covariant form. We find that the major symmetry condition relates the Lie symmetries with the special projective symmetries of the connection. A simialr result has been obtained by Prince and Crampin in [7] using the bundle formulation of second order ODEs. In section 5 we apply these conditions in the case of Riemannian spaces. We solve the

\footnotetext{
*Email: mtsampa@phys.uoa.gr

†Email: paliathanasis@gmail.com
} 
symmetry conditions and in Theorem 1 we give the Lie symmetry vectors in terms of the collineations of the metric. In section 6 we apply Theorem 1 to the case of spaces of constant curvature of dimension $n$ and compute the complete set of Lie symmetries of the system of affinely parameterized geodesic equations in these spaces. We distinguish the case of the flat space and the spaces of non-zero curvature. Finally we prove the validity of the conjecture made in [6].

\section{Preliminary results}

Consider a $C^{\infty}$ manifold $M$ of dimension $n$, endowed with a symmetric ${ }^{1}$ connection [8].

Let $X^{a}$ be a vector field on the manifold. The connection defines a family of curves on the manifold, called autoparallels (or paths), by the requirement that the covariant derivative of the tangent to these curves is parallel to itself, that is:

$$
\nabla_{\dot{x}(t)} \dot{x}(t)=\phi(t) \dot{x}(t)
$$

where $t$ is a parameter along the curves. When the parametrization is such that $\phi$ vanishes we say that the autoparallel is affinely parameterized and in this case $t$ is called an affine parameter.

In a local coordinate system $\left\{x^{i} \mid i=1, \ldots, n\right\}$ equation (1) is written as the system of ODEs:

$$
\ddot{x}^{i}(t)+\Gamma_{j k}^{i}(x(t)) \dot{x}^{j}(t) \dot{x}^{k}(t)=\phi(t) \dot{x}^{i}(t), \quad i=1, \ldots, n
$$

where $\Gamma_{j k}^{i} \partial_{i}=\nabla_{j} \partial_{k}$. The Lie derivative (of a not-necessarily symmetric connection) $\Gamma_{j k}^{i}$ with respect to a vector field $\mathbf{X}=X^{a} \partial_{a}$ is defined as follows (see Yano [9] equation (2.16)):

$$
\mathcal{L}_{X} \Gamma_{j k}^{i}=X_{, j k}^{i}+\Gamma_{j k, l}^{i} X^{l}+X_{, k}^{l} \Gamma_{l j}^{i}+X_{, j}^{l} \Gamma_{l k}^{i}-X_{, l}^{i} \Gamma_{j k}^{l}
$$

We say that the vector field $\mathbf{X}$ is an affine collineation or affine motion iff

$$
\mathcal{L}_{X} \Gamma_{j k}^{i}=0
$$

In flat space equation (4) implies the condition:

$$
X_{a, b c}=0
$$

whose solution is:

$$
X_{a}=B_{a b} x^{b}+C_{a}
$$

where $B_{a b}, C_{a}$ are constants. The geometric property/definition of affine collineations is that they preserve the set of autoparallels of the connection together with their affine parametrization (that is, by an affine symmetry an affinely parameterized autoparallel goes over to an affinely parameterized autoparallel of the same connection). From (6) we infer that in an n-dimensional space there are at most $n+n^{2}=n(n+1)$ Affine Collineation Vectors and when this is the case, it can be shown that the space is flat.

Note that the equation of the autoparallels for affine parametrization takes the form:

$$
\ddot{x}^{i}(t)+\Gamma_{j k}^{i}(x(t)) \dot{x}^{j}(t) \dot{x}^{k}(t)=0, \quad i=1, \ldots, n .
$$

We say that a vector field $\mathbf{X}$ is a projective collineation of the connection if there exists a one form $f_{i}$ so that the following condition holds:

$$
\mathcal{L}_{X} \Gamma_{j k}^{i}=f_{j} \delta_{k}^{i}+f_{k} \delta_{j}^{i}
$$

In Riemannian space the one form is closed and $f_{i}$ is replaced with $f_{, i}$ where $f\left(x^{i}\right)$ is a smooth function.

In flat space condition (8) implies that:

$$
X_{a, b}=B_{a b}+\left(A_{c} x^{c}\right) g_{a b}+C_{b} x_{a}
$$

which has the solution:

$$
X_{a}=B_{a b} x^{b}+\left(A_{b} x^{b}\right) x_{a}+C_{a}
$$

${ }^{1}$ The coefficients $\Gamma_{j k}^{i}$ in general are not symmetric. In the autoparallel equation (1) the antisymmetric part of $\Gamma_{j k}^{i}$ (the torsion) does play a role. 
where again the various coefficients are constants. In an $\mathrm{n}$ - dimensional space there are at most $n^{2}+n+n=$ $n(n+2)$ projective collineations of the connection and when this is the case, it can be shown that the space is flat. This holds in any space irrespective of the signature of the metric and the (finite) dimension of space.

The geometric property/definition of the (proper) projective collineations is that they preserve the set of autoparallels, but they do not preserve the affine parametrization.

\section{Lie point symmetries of the autoparallel equations}

We consider the system of ODEs (2) and determine its Lie point symmetries generated by the vector field $X$. Because the ODEs are of second order, we must prolong $X$ up to the second prolongation. Let $G^{[1] i}$ be the coefficients of the first prolongation. From the prolongation formula we have

$$
G^{[1] i}=\frac{d}{d t} \eta^{i}-\dot{x}^{i} \frac{d}{d t} \xi=\eta_{, t}^{i}+\eta_{, j}^{i} \dot{x}^{j}-\xi_{, t} \dot{x}^{i}-\xi_{, j} \dot{x}^{i} \dot{x}^{j}
$$

For the second prolongation coefficient $G^{[2] i}$ of $X$ we have:

$$
\begin{aligned}
G^{[2] i} & =\frac{d}{d t} G^{[1] i}-\ddot{x}^{i} \frac{d}{d t} \xi \\
& =\eta_{, t t}^{i}+2 \eta_{, t j}^{i} \dot{x}^{j}+\eta_{, k j}^{i} \dot{x}^{k} \dot{x}^{j}-\xi_{, t t} \dot{x}^{i}-2 \xi_{, t j} \dot{x}^{j} \dot{x}^{i}-\xi_{j k} \dot{x}^{j} \dot{x}^{k} \dot{x}^{i} \\
& +\eta_{, j}^{i} \ddot{x}^{j}-2 \xi_{,{ }^{\prime}} \ddot{x}^{i}-\xi_{, j} \ddot{x}^{j} \dot{x}^{i}-2 \xi_{, j} \ddot{x}^{i} \dot{x}^{j} .
\end{aligned}
$$

The computation of Lie symmetries can be done in two different ways : Either by using the linear operator $\mathbf{A}$ associated with the system of ODEs and demanding the symmetry condition $\left[X^{[1]}, \mathbf{A}\right]=\lambda \mathbf{A}$, or by computing directly the action of the prolonged generator on the equation and demanding that $X^{[1]}(O D E)=0 \mathrm{mod}$ $(O D E=0)$ where ODE is given by $(2)$.

\section{Calculation of Lie symmetries using the associated linear operator}

We write the system of ODEs in the form $\ddot{x}^{i}=\omega^{i}(x, \dot{x}, t)$ where:

$$
\omega^{i}(x, \dot{x}, t)=-\Gamma_{j k}^{i}(x) \dot{x}^{j} \dot{x}^{k}-\phi(x) \dot{x}^{i} .
$$

The associated linear operator defined by this system of ODEs is:

$$
\mathbf{A}=\frac{\partial}{\partial t}+\dot{x}^{i} \frac{\partial}{\partial x^{i}}+\omega^{i}\left(t, x^{j}, \dot{x}^{j}\right) \frac{\partial}{\partial \dot{x}^{i}} .
$$

The condition for a Lie symmetry for the system of equations is [10]:

$$
\left[X^{[1]}, \mathbf{A}\right]=\lambda\left(x^{a}\right) \mathbf{A}
$$

where $X^{[1]}$ is the first prolongation of the symmetry vector $X=\xi(t, x) \partial_{t}+\eta^{i}(t, x) \partial_{x^{i}}$ defined as follows:

$$
X^{[1]}=\xi(t, x, \dot{x}) \partial_{t}+\eta^{i}(t, x, \dot{x}) \partial_{x^{i}}+G^{[1] i} \partial_{\dot{x}^{i}} .
$$

It is a standard result [10] that (14) leads to the following three conditions:

$$
\begin{aligned}
-\mathbf{A} \xi & =\lambda \\
G^{[1] i} & =\mathbf{A} \eta^{i}-\dot{x}^{i} \mathbf{A} \xi \\
X^{[1]}\left(\omega^{i}\right)-\mathbf{A}\left(G^{[1] i}\right) & =-\omega^{i} \mathbf{A} \xi .
\end{aligned}
$$


For any function $f\left(t, x^{i}\right)$ the $\mathbf{A} f=\frac{d f}{d t}$ where $\frac{d f}{d t}=f_{, t}+f_{, i} \dot{x}^{i}$ is the total derivative of $f$. Using this result we write the symmetry conditions as follows:

$$
\begin{aligned}
\lambda & =-\frac{d \xi}{d t} \\
G^{[1] i} & =\frac{d \eta^{i}}{d t}-\dot{x}^{i} \frac{d \xi}{d t} \\
X^{[1]}\left(\omega^{i}\right)-A\left(G^{[1] i}\right) & =-\omega^{i} \frac{d \xi}{d t} .
\end{aligned}
$$

We note that the second condition (20) defines the first prolongation $G^{[1] i}$. The first equation (19) gives the factor $\lambda$, therefore the essential symmetry condition is equation (21).

To compute the symmetry condition first we have to compute the quantities $X^{[1]}\left(\omega^{i}\right)$ and $A\left(G^{[1] i}\right)-\omega^{i} \frac{d \xi}{d t}$ taking into consideration (20) and (12). The result of this formal calculation is:

$$
\begin{aligned}
X^{[1]}\left(\omega^{i}\right) & =\left(\xi \partial_{t}+\eta^{i} \partial_{x^{i}}+G^{[1] i} \partial_{\dot{x}^{i}}\right)\left(-\Gamma_{j k}^{i}(x) \dot{x}^{j} \dot{x}^{k}-\phi(x) \dot{x}^{i}\right) \\
& =-\eta_{, t}^{i} \phi \\
+ & \left(-\xi \phi{ }_{, t} \delta_{j}^{i}-\phi_{, k} \eta^{k} \delta_{j}^{i}-\eta_{,{ }^{k}}^{k} \Gamma_{k j}^{i}-\eta_{, t}^{k} \Gamma_{j k}^{i}-\phi \eta_{, j}^{i}+\phi \xi_{, t} \delta_{j}^{i}\right) \dot{x}^{j} \\
+ & \left(-\xi \Gamma_{(k j), t}^{i}-\eta^{l} \Gamma_{(k j), l}^{i}-\eta_{, k}^{l} \Gamma_{(l j)}^{i}-\eta_{, k}^{l} \Gamma_{(j l)}^{i}+\phi \xi_{, k} \delta_{j}^{i}+2 \xi_{, t} \Gamma_{(k j)}^{i}\right) \dot{x}^{j} \dot{x}^{k} \\
+ & \xi_{,(k} \Gamma_{j l)}^{i} \dot{x}^{j} \dot{x}^{k} \dot{x}^{l} . \\
A\left(G^{[1] i}\right)-\omega^{i} \frac{d \xi}{d t}=\eta_{, t t}^{i} & \left(2 \eta_{, t j}^{i}+\phi \eta_{, j}^{i}-2 \phi \xi_{, t} \delta_{j}^{i}-\xi_{, t t} \delta_{j}^{i}\right) \dot{x}^{j} \\
& \quad+\left(\eta_{,(j k)}^{i}-2 \xi_{, t(j} \delta_{k)}^{i}-\eta_{, l}^{i} \Gamma_{(j k)}^{l}+2 \xi_{, t} \Gamma_{(j k)}^{i}-2 \phi \xi_{(, j} \delta_{k)}^{i}\right) \dot{x}^{j} \dot{x}^{k} \\
& \quad+\left(\xi_{,(j} \Gamma_{k l)}^{i}+\xi_{, m} \Gamma_{(k l}^{m} \delta_{j)}^{i}-\xi_{,(j k} \delta_{l)}^{i}\right) \dot{x}^{j} \dot{x}^{k} \dot{x}^{l} .
\end{aligned}
$$

Substituting in the symmetry condition (21) and collecting terms of the same order in $\dot{x}^{j}$ we find the following equations: $(i=1, \ldots, n)$ :

$(\dot{x})^{0}$ terms:

$$
\eta_{, t t}^{i}+\eta_{, t}^{i} \phi=0
$$

$(\dot{x})^{1}$ terms:

$$
\xi_{, t t} \delta_{j}^{i}-\xi \phi_{, t} \delta_{j}^{i}-2\left[\eta_{, t j}^{i}+\eta_{, t}^{k} \Gamma_{(k j)}^{i}\right]-\left[\phi \xi_{, t}+\phi_{, k} \eta^{k}\right] \delta_{j}^{i}=0
$$

$(\dot{x})^{2}$ terms:

$$
\begin{gathered}
\left(-\eta_{,(j k)}^{i}-\eta^{l} \Gamma_{(j k), l}^{i}-\eta_{, k}^{l} \Gamma_{l j}^{i}-\eta_{, k}^{l} \Gamma_{j l}^{i}+\eta_{, l}^{i} \Gamma_{j k}^{l}\right)+2 \xi_{, t(j} \delta_{k)}^{i}-2 \phi \xi_{(, j} \delta_{k)}^{i}-\xi \Gamma_{(k j), t}^{i}=0 \quad \Rightarrow \\
\mathcal{L}_{\boldsymbol{\eta}} \Gamma_{(j k)}^{i}=-2 \phi \xi_{(, j} \delta_{k)}^{i}+\xi \Gamma_{(k j), t}^{i}+2 \xi_{, t(j} \delta_{k)}^{i}
\end{gathered}
$$

$(\dot{x})^{3}$ terms:

$$
\left(\xi_{,(j k}-\xi_{,|e|} \Gamma_{(j k}^{e}\right) \delta_{l)}^{i}=0
$$

Define the quantity:

$$
\Phi=\xi_{, t}-\phi \xi
$$

Then condition (25) is written (note that $\phi,,_{i}=0$ ):

$$
\mathcal{L}_{\boldsymbol{\eta}} \Gamma_{(j k)}^{i}=2 \Phi_{(, j} \delta_{k)}^{i}-\xi \Gamma_{(k j), t}^{i} .
$$

If we consider the vector $\xi=\xi \partial_{t}$ (which does not have components along $\partial_{i}$ ) we compute that:

$$
\mathcal{L}_{\xi} \Gamma_{(j k)}^{i}=\xi \Gamma_{(k j), t}^{i}
$$


hence (28) is written:

$$
\mathcal{L}_{\mathbf{X}} \Gamma_{(j k)}^{i}=2 \Phi_{(, j} \delta_{k)}^{i}
$$

where $\mathbf{X}=\xi+\eta=\xi \partial_{t}+\eta^{i}(t, x) \partial_{x^{i}}$. We observe that this condition is precisely condition (8) for a projective collineation of the connection $\Gamma_{(j k)}^{i}$ along the symmetry vector $\mathbf{X}$ and with projecting function $\Phi$. Concerning the other conditions we note that $(24)$ can be written in covariant form (relevant to the indices $a=1,2, \ldots, n$ ) as follows:

$$
\Phi_{, t} \delta_{j}^{i}-2 \eta_{, t \mid j}^{i}=0
$$

where $\eta_{, t \mid j}^{i}=\eta_{, t j}^{i}+\eta_{, t}^{k} \Gamma_{(k j)}^{i}$ is the covariant derivative with respect to $\Gamma_{(k j)}^{i}$ of the vector $\eta_{, t}^{i}$. Similarly condition (26) can be written:

$$
\xi_{\mid(j k} \delta_{l)}^{i}=0
$$

Contracting the indices $i, l$ we find the final form:

$$
\xi_{\mid(j k)}=0
$$

This implies that $\xi,{ }_{i}$ is a gradient $\mathrm{KV}$ of the metric of the space $x^{i}$.

Condition (23) is obviously in covariant form with respect to the indices $a$.

We arrive at the following conclusion:

1. The conditions for the Lie symmetries of the autoparallel equations (12) (not necessarily affinely parameterized) for a general connection defined on a $C^{\infty}$ manifold are the following:

$$
\begin{aligned}
\eta_{, t t}^{i}+\eta_{, t}^{i} \phi & =0 \\
\xi_{\mid(j k)} & =0 \\
\Phi_{, t} \delta_{j}^{i}-2 \eta_{, t \mid j}^{i} & =0 \\
\mathcal{L}_{\mathbf{X}} \Gamma_{(j k)}^{i} & =2 \Phi_{(, j} \delta_{k)}^{i} .
\end{aligned}
$$

These are covariant equations because if we consider the connection in the augmented $n+1$ space $\left\{x^{i}, t\right\}$, all components of $\Gamma$ which contain an index along the direction of $t$ vanish, therefore the partial derivatives with respect to $t$ can be replaced with covariant derivative with respect to $t$.

2. Equation (32) gives the functional dependence of $\eta^{i}$ on $t$ and the non-affine parametrization function $\phi(t)$.

3. Equation (33) gives that the vector $\xi_{, i}$ is a gradient constant (Killing in the case of a Riemannian space) vector of the $n$-dimensional space $\left\{x^{i}\right\}$.

4. Equation (34) relates the functional dependence of $\eta^{i}$ and $\xi$ in terms of $t$.

5. Equation (35) is the most important equation for our purpose, because it states that the symmetry vector $X$ is a projective collineation in the jet space $\left\{t, x^{i}\right\}$. In the space $\left\{x^{i}\right\}$ the vectors $\eta^{i}(t, x) \partial_{x^{i}}$ are also projective collineations.

In the following we restrict our considerations to the case of Riemannian connections that is the $\Gamma_{j k}^{i}$ are symmetric and the covariant derivative of the metric vanishes.

\section{Calculation of the symmetry vector for a Riemannian connection}

We compute the Lie symmetry vectors for the case of affine parametrization $(\phi=0)$ and the assumption $\Gamma_{j k, t}^{i}=0$ i.e. the $\Gamma_{j k}^{i}$ are independent of the parameter $t$. The later is a reasonable assumption because the 
$\Gamma_{j k}^{i}$ are computed in terms of the metric which does not depend on the parameter $t$. Under these assumptions the symmetry conditions (32) - (35) read:

$$
\begin{aligned}
\eta_{, t t}^{i} & =0 \\
\xi_{\mid(j k)} & =0 \\
\xi_{, t t} \delta_{j}^{i}-2 \eta_{, t \mid j}^{i} & =0 \\
\mathcal{L}_{\boldsymbol{\eta}} \Gamma_{j k}^{i} & =2 \xi_{, t(j} \delta_{k)}^{i} .
\end{aligned}
$$

We proceed with the solution of this system of equations (see also [7] Table II).

Equation (36) implies:

$$
\eta^{i}(t, x)=A^{i}(x) t+B^{i}(x)
$$

where $A^{i}(x), B^{i}(x)$ are arbitrary differentiable vector fields.

The solution of equation (37) is:

$$
\xi(t, x)=C_{J}(t) S^{J}(x)+D(t)
$$

where $C_{J}(t), D(t)$ are arbitrary functions of the affine parameter $t$ and $S^{J}(x)$ is a function whose gradient is a gradient $\mathrm{KV}$ i.e.:

$$
S^{J}(x)_{\mid(i, j)}=0
$$

The index $J$ runs through the number of gradient KVs of the metric. Condition (38) gives:

$$
2 A(x)_{\mid j}^{i}=\left[C_{J}(t), t t S^{J}(x)+D(t), t t\right] \delta_{j}^{i} .
$$

Because the left hand side is a function of $x$ only we must have:

$$
\begin{aligned}
D(t), t t & =M \Rightarrow D(t)=\frac{1}{2} M t^{2}+K t+L \text { where } M, K, L \text { constants } \\
C_{J}(t), t t & =G_{J}=\text { constant } \Rightarrow C_{J}(t)=\frac{1}{2} G_{J} t^{2}+E_{J} t+F_{J} \text { where } G_{J}, E_{J}, F_{J} \text { constants. }
\end{aligned}
$$

Replacing in (43) we find:

$$
2 A(x)_{; j}^{i}=\left(G_{J} S^{J}(x)+M\right) \delta_{j}^{i} \Rightarrow A(x)_{i ; j}=\frac{1}{2}\left(G_{J} S^{J}(x)+M\right) g_{i j}
$$

where we have lowered the index because the connection is metric (i.e. $g_{i j \mid k}=0$ ). The last equation implies that the vector $A(x)^{i}$ is a conformal Killing vector with conformal factor $\psi=\frac{1}{2}\left(G_{J} S^{J}(x)+M\right)$. Because $A(x)_{[i ; j]}=0$ this vector is a gradient CKV.

We continue with condition (39) and replace $\eta^{i}(t, x)$ from (40):

$$
\begin{aligned}
\mathcal{L}_{\mathbf{A}} \Gamma_{j k}^{i} t+\mathcal{L}_{\mathbf{B}} \Gamma_{j k}^{i} & =2 \xi, t,{ }_{j} \delta_{k)}^{i}=2\left[\left(G_{J} t+E_{J}\right) S^{J}(x)+M t+K\right]_{\mid(j} \delta_{k)}^{i}=2\left(G_{J} t+E_{J}\right) S^{J}(x)_{\mid(j} \delta_{k)}^{i} \Rightarrow \\
\mathcal{L}_{\mathbf{A}} \Gamma_{j k}^{i} & =2 G_{J} S^{J}(x)_{,(j} \delta_{k)}^{i} \\
\mathcal{L}_{\mathbf{B}} \Gamma_{j k}^{i} & =2 E_{J} S^{J}(x)_{,(j} \delta_{k)}^{i} .
\end{aligned}
$$

The last two equations imply that the vectors $A^{i}(x), B^{i}(x)$ are special projective collineations or affine collineations of the metric - or one of their specializations - with projective functions $G_{J} S^{J}(x)$ and $E_{J} S^{J}(x)$ or zero respectively. Note that relations (47), (48) remain true if we add a $\mathrm{KV}$ to the vectors $A^{i}(x), B^{i}(x)$, therefore these vectors are determined up to a $\mathrm{KV}^{2}$.

It is well known that in a Riemannian space a CKV $K^{i}$ with conformal factor $\psi\left(x^{i}\right)$ satisfies the identity:

$$
\mathcal{L}_{\mathbf{K}} \Gamma_{(j k)}^{i}=g^{i s}\left[\psi_{, j} g_{k s}+\psi_{, k} g_{j s}-\psi_{, s} g_{j k}\right]
$$

Applying this identity to the $\mathrm{CKV} A^{i}$ we find:

$$
G_{J} S^{J}(x)_{, k}=0 \Rightarrow G_{J} S^{J}(x)=2 \rho=\text { constant. }
$$

\footnotetext{
${ }^{2}$ Because $A^{i}$ is a projective collineation and a CKV it must be a HKV.
} 
This implies that $A^{i}$ is a gradient $H K V$ with homothetic factor $\rho+\frac{1}{2} M$ (not necessarily proper). Furthermore (46) implies:

$$
\begin{aligned}
2 A^{i} & =(2 \rho+M) x^{i}+2 L^{i} \Rightarrow \\
A^{i} & =\left(\rho+\frac{1}{2} M\right) x^{i}+L^{i}
\end{aligned}
$$

where $L^{i}$ is a non-gradient $\mathrm{KV}$.

We continue with the special projective collineation vector $B^{i}$. For this vector we use the property that for a symmetric connection the following identity holds:

$$
\mathcal{L}_{\mathbf{B}} \Gamma_{(j k)}^{i}=B_{; j k}^{i}-R_{j k l}^{i} B^{l} .
$$

Replacing the left hand side from (48) we find:

$$
B_{; j k}^{i}-R_{j k l}^{i} B^{l}=2 E_{J} S^{J}(x),{ }_{(j} \delta_{k)}^{i} .
$$

Contracting the indices $i, j$ we find:

$$
\left(B_{; i}^{i}-(n+1) E_{J} S^{J}(x)\right)_{; k}=0
$$

which implies:

$$
B_{; i}^{i}=(n+1) E_{J} S^{J}(x)+2 b
$$

where $b=$ constant. In case this vector is an affine collineation then $B_{; i}^{i}=2 b$. Using the above results we find for $\xi(t, x)$ :

$$
\begin{aligned}
\xi(t, x) & =C_{J}(t) S^{J}+D(t) \\
& =\left(\frac{1}{2} G_{J} t^{2}+E_{J} t+F_{J}\right) S^{J}+\frac{1}{2} M t^{2}+K t+L \\
& =\frac{1}{2}\left(G_{J} S^{J}+M\right) t^{2}+\left(E_{J} S^{J}+K\right) t+F_{J} S^{J}+L
\end{aligned}
$$

We summarize the above results in the following Theorem

Theorem 1 The Lie symmetry vector $\mathbf{X}=\xi+\eta=\xi(t, x) \partial_{t}+\eta^{i}(t, x) \partial_{x^{i}}$ of the equation of geodesics ( 7$)$ in a Riemannian space involves all symmetry vectors, that is, (gradient) KVs, (gradient) HKVs, special PCs and their degeneracies ACs, HKV and KVs as follows:

A. The metric admits gradient KVs. Then:

a. The function:

$$
\xi(t, x)=\left(\rho+\frac{1}{2} M\right) t^{2}+\left[E_{J} S^{J}+K\right] t+F_{J} S^{J}+L
$$

where $\rho, M, b, K, F_{J}, L$ are constants, the index $J$ running along the number of gradient $K V$.

$b$. The vector:

$$
\eta^{i}(t, x)=A^{i}(x) t+B^{i}(x)+D^{i}(x)
$$

where the vector $A^{i}(x)$ is a gradient $H K V$ with conformal factor $\psi=\rho+\frac{1}{2} M, D^{i}(x)$ is a non-gradient $K V$ of the metric and $B^{i}(x)$ is either a special projective collineation with projection function $E_{J} S^{J}(x)$ whose divergence $B_{; i}^{i}$ satisfies condition (54) or an $A C$ and $E_{J}=0$ in (55).

$B$. The metric does not admit gradient KVs. Then :

a. The function:

$$
\xi(t, x)=\frac{1}{2} M t^{2}+K t+L
$$

b. The vector:

$$
\eta^{i}(t, x)=A^{i}(x) t+B^{i}(x)+D^{i}(x)
$$

where $A^{i}(x)$ is a gradient $H K V$ with conformal factor $\psi=\frac{1}{2} M, D^{i}(x)$ is a non-gradient $K V$ of the metric and $B^{i}(x)$ is an $A C$. If further the metric does not admit gradient $H K V$ then:

$$
\begin{aligned}
\xi(t) & =K t+L \\
\eta^{i}(x) & =B^{i}(x)+D^{i}(x) .
\end{aligned}
$$




\section{The case of spaces of constant curvature}

The metric of a space of constant curvature $K=0, \pm 1$ and dimension $n$ in Cartesian (stereographic) coordinates is:

$$
d s^{2}=U\left(x^{i}\right) \eta_{i j} d x^{i} d x^{j}
$$

where $U\left(x^{i}\right)=\frac{1}{1+\frac{K x \cdot x}{4}}, \eta_{i j}=\operatorname{diag}( \pm 1, \pm 1, \ldots, \pm 1)$. It is easy to show that the connection coefficients of this metric are:

$$
\Gamma_{j k}^{i}=-\frac{K U}{2}\left(x_{k} \delta_{j}^{i}+x_{j} \delta_{k}^{i}-x^{i} \eta_{j k}\right)
$$

hence the geodesic equations for affine parametrization are:

$$
\ddot{x}^{i}-\frac{K U}{2}\left(2 x_{k} \dot{x}^{k} \dot{x}^{i}-\left(\eta_{j k} \dot{x}^{j} \dot{x}^{k}\right) \dot{x}^{i}\right)=0
$$

where a dot indicates derivation with respect to the affine parameter $s$ (say). We shall apply Theorem 1 to determine the Lie symmetries of the geodesic equations (63).

In order to apply Theorem 1 we need to know the conformal and the projective collineations of spaces of constant curvature. From the literature we have the following results $(I, J=1, \ldots, n)$.

A. Flat spaces

a. Conformal algebra

$\frac{n(n-1)}{2}$ Non-gradient Killing vectors:

$$
\mathbf{X}_{I J}=\delta_{[I}^{j} \delta_{J]}^{i} x_{j} \partial_{i}
$$

$n$ Gradient Killing vectors:

$$
\mathbf{Y}_{I}=\delta_{I}^{i} \partial_{i}
$$

1 Gradient Homothetic Killing vector:

$$
\mathbf{H}=x^{i} \partial_{i} \text { with homothety factor } \psi=1
$$

$n$ non-gradient special CKVs:

$$
\mathbf{C}_{I}=\left[2 x_{I} x^{i}-\delta_{I}^{i}\left(x^{k} x_{k}\right)\right] \partial_{i} \text { with conformal factor } \psi=2 x^{i}
$$

b. $n(n+1)$ Proper Affine Collineations[9]:

$$
\mathbf{A}_{j}^{i}=\left[b_{j}^{i} x^{j}+c^{i}\right] \partial_{i}
$$

c. $n(n+2)$ Special Projective Collineations[11]:

$$
\left[\left(c_{j} x^{j}\right) x^{i}+b_{j}^{i} x^{j}+a^{i}\right] \partial_{i} .
$$

B. Spaces of constant non-vanishing curvature (the indices $I, J$ count vectors):

a. Conformal algebra[12]:

$n$ Non-gradient Killing vectors:

$$
\mathbf{Y}_{I}=\frac{1}{U}\left[(2 U-1) \delta_{I}^{i}+\frac{K U}{2} x_{I} x^{i}\right] \partial_{i}
$$

$\frac{n(n-1)}{2}$ Non-gradient Killing vectors:

$$
\mathbf{X}_{I J}=\delta_{[I}^{j} \delta_{J]}^{i} \partial_{i}
$$

1 Gradient Conformal Killing vector:

$$
\mathbf{H}=x^{i} \partial_{i}
$$


$n$ Gradient Conformal Killing vectors:

$$
\mathbf{X}_{I}=\frac{1}{U}\left[\delta_{I}^{i}-\frac{1}{2} K U x_{I} x^{i}\right] \partial_{i}
$$

b. Proper Affine Collineations[13]:

Not admitted.

c. Proper Special Projective Collineations ${ }^{3}$ :

Not admitted.

\subsection{The case of flat space}

In this case the equation of geodesics when affinely parameterized is:

$$
\ddot{x}^{i}=0 i=1,2 .,, n \text {. }
$$

We know that these equations admit the maximal number of Lie symmetries. We determine these symmetries using Theorem 1. The gradient KVs $\delta_{I}^{i} \partial_{i}$ imply:

$$
S^{J}(x)=\delta_{i}^{J} x^{i}
$$

(plus an irrelevant constant). The gradient homothetic vector $x^{i} \partial_{i}$ implies:

$$
A^{i}=x^{i}, L^{i}=0 \text {. }
$$

and the homothetic factor $\psi=1$ :

$$
\rho+\frac{1}{2} M=1
$$

Equation (46) gives:

$$
2 \delta_{j}^{i}=\left(G_{J} S^{J}(x)+M\right) \delta_{j}^{i} \Rightarrow G_{J}=0, M=2 .
$$

Condition (54) on the proper projective collineations gives:

$$
b_{; i}^{i}+(n+1)\left(c_{i} x^{i}\right)=(n+1) E_{i} x^{i}+2 b \Rightarrow E_{i}=c_{i}, b=\frac{1}{2} b_{i}^{i} .
$$

We have then from (55) for the symmetry function $\xi(t, x)$ :

$$
\xi(t, x)=t^{2}+\left(c_{i} x^{i}+K\right) t+F_{i} x^{i}+L
$$

where $K, F_{i}, L$ are constants.

Concerning the symmetry vector $n^{i}$ we have form (56):

$$
n^{i}=x^{i} t+\left[\left(c_{j} x^{j}\right) x^{i}+b_{j}^{i} x^{j}+a^{i}\right]+\delta_{[I}^{j} \delta_{J]}^{i} x_{j}+\delta_{I}^{i} .
$$

These symmetry quantities must be a solution of the symmetry conditions (36) - (39). It is an easy exercise to show that this is the case indeed. Concerning (39) we find:

$$
\mathcal{L}_{\boldsymbol{\eta}} \Gamma_{j k}^{i}=2 c_{(j} \delta_{k)}^{i}=n_{; j k}^{i}
$$

which is correct. Therefore the solution we have found satisfies the Lie symmetry conditions.

\footnotetext{
${ }^{3}$ A space of constant curvature admits $n(n+2)$ Proper Projective Collineations[13]:

$$
\phi, i+D_{i}(n \geq 2)
$$

where $\phi=c+\frac{\left(1-K r^{2} / 4\right) a_{i} x^{i}+b_{i j} x^{i} x^{j}}{\left(1-K r^{2} / 4\right)^{2}} a_{i}, b_{i j}=b_{j i}, c$ and $D_{i}$ is a Killing vector. The constant $c$ is irrelevant because it does not give a proper projective collineation. These collineations are not special.
} 


\subsection{The case of a space of constant non-vanishing curvature}

In this case the system whose Lie symmetries we want to find are geodesic equations (63) of a metric of constant non-vanishing curvature. These metrics do not have gradient $\mathrm{KV}$ s therefore $S^{J}(x)=0$ and the index $J$ is suppressed. Furthermore they do not admit a gradient homothetic vector, (proper) Special PCs and ACs. Therefore the last case of the Theorem applies and we have from (60) and (59) and (64), (65):

$$
\begin{aligned}
\xi(t) & =K t+L \\
n^{i}(x) & =\delta_{\left[I^{\prime}\right.}^{j} \delta_{\left.J^{\prime}\right]}^{i} x_{j}+\frac{1}{U}\left[(2 U-1) \delta_{K^{\prime}}^{i}+\frac{K U}{2} x_{I^{\prime}} x^{i}\right] \text { where } I^{\prime}, J^{\prime}, K^{\prime}=1, \ldots . . .
\end{aligned}
$$

i.e. $n^{i}(x)$ contains only the non-gradient KVs. It is easy to show that these quantities satisfy all the conditions (36) - (39) i.e. they are Lie symmetries of the geodesic equations (63). We conclude that in this case we have $\frac{n(n+1)}{2}+2$ symmetry vectors (as many as the parameters $\left.I^{\prime}, J^{\prime}, K^{\prime}, K, L\right)$. If one applies the general results to the various cases considered in [6] one recovers their results.

Finally it follows that the conjecture made in [6], claiming that the Lie symmetries of the geodesics of the maximally symmetric spaces of non-vanishing curvature are only the KVs, is true. Another verification of this conjecture is given in [14].

\subsection{The Lie symmetries of geodesic equations in an Einstein space}

Spaces of constant curvature are Einstein spaces whose curvature scalar is a constant. In this section we generalize the results of last section to proper Einstein spaces in which $R$ is not a constant. Suppose $X^{a}$ is a projective collineation with projection function $\phi\left(x^{a}\right)$, so that $\mathcal{L}_{X} \Gamma_{b c}^{a}=\phi_{, b} \delta_{c}^{a}+\phi_{, c} \delta_{b}^{a}$. For a proper Einstein space $(R \neq 0)$ we have $R_{a b}=\frac{R}{n} g_{a b}$ from which follows:

$$
\mathcal{L}_{X} g_{a b}=\frac{n(1-n)}{R} \phi_{; a b}-\mathcal{L}_{X}(\ln R) g_{a b}
$$

Using the contracted Bianchi identity $\left(R^{i j}-\frac{1}{2} R g^{i j}\right)_{; j}=0$ it follows that in an Einstein space of dimension $n>2$ the curvature scalar $R=$ constant and (68) reduces to:

$$
\mathcal{L}_{X} g_{a b}=\frac{n(1-n)}{R} \phi_{; a b}
$$

It follows that if $X^{a}$ generates either an affine or a special projective collineation then $\phi_{; a b}=0$ hence $X^{a}$ reduces to a KV. This means that proper Einstein spaces do not admit affine collineations or special projective collineations.

A proper Einstein space admits in general a gradient HKV. Indeed if $A^{a}$ is a HKV in a proper Einstein space, then $\mathcal{L}_{X} R_{a b}=0$ and using the contracted Bianchi identity we find:

$$
\mathcal{L}_{A} g_{a b}=\frac{1}{n} A(\ln R) g_{a b}
$$

If the conformal factor of $A^{a}$ is $\psi$ (a constant) then:

$$
A(\ln R)=\psi n \Rightarrow(\ln R)^{, a}=\psi n A^{a} \Rightarrow A^{a}=\left(\frac{1}{\psi n} \ln R\right)^{, a}
$$

that is $A^{a}$ is a gradient HKV.

The above results and Theorem 1 lead to the following conclusion:

Theorem 2 The Lie symmetries of the geodesic equations in a proper Einstein space of curvature scalar $R$ $(\neq 0)$ are given by the vectors $\mathbf{X}=\xi+\eta=\xi(t, x) \partial_{t}+\eta^{i}(t, x) \partial_{x^{i}}$ as follows:

A. The metric admits gradient $K V s S^{J}\left(x^{a}\right)$. Then :

a. The function:

$$
\xi(t, x)=\left(\rho+\frac{1}{2} M\right) t^{2}+\left[E_{J} S^{J}+K\right] t+F_{J} S^{J}+L
$$


where $\rho, M, b, K, F_{J}, L$ are constants, the index $J$ running along the number of gradient $K V$.

b. The vector:

$$
\eta^{i}(t, x)=A^{i}(x) t+D^{i}(x)
$$

where the vector $A^{a}$ is a gradient $H K V$ with conformal factor $\rho+\frac{1}{2} M=\frac{1}{n} A(\ln R)$ and $D^{i}(x)$ is a non-gradient $K V$ of the metric

B. The metric does not admit gradient KVs. Then :

a. The function:

$$
\xi(t, x)=\frac{1}{2} M t^{2}+K t+L
$$

b. The vector:

$$
\eta^{i}(t, x)=A^{i}(x) t+D^{i}(x)
$$

where $A^{i}(x)$ is a gradient $H K V$ with conformal factor $\frac{1}{2} M=\frac{1}{n} A(\ln R)$ (if admitted) and $D^{i}(x)$ is a non-gradient $K V$.

Theorem 2 extends and amends the conjecture of [6] to the more general case of Einstein spaces.

\section{Examples}

\subsection{The geodesic symmetries of de Sitter spacetime}

To show the use of Theorem 1 in practice we consider the symmetries of geodesics in de Sitter spacetime, which is is a hyperbolic space of constant curvature. The metric of the space is:

$$
d s^{2}=\frac{1}{\left(1+\frac{K}{4}\left(-t^{2}+x^{2}+y^{2}+z^{2}\right)\right)^{2}}\left(-d t^{2}+d x^{2}+d y^{2}+d z^{2}\right) .
$$

The $10 \mathrm{KVs}$ are

$$
\begin{aligned}
& X_{1}=(-x t) \partial_{t}+\left(\frac{\left(-t^{2}-x^{2}+y^{2}+z^{2}\right)}{2}-\frac{2}{K}\right) \partial_{x}+(-y x) \partial_{y}+(-z x) \partial_{x} \\
& X_{2}=(y t) \partial_{t}+(y x) \partial_{x}+\left(\frac{\left(-x^{2}-z^{2}+y^{2}+t^{2}\right)}{2}+\frac{2}{K}\right) \partial_{y}+(y z) \partial_{x} \\
& X_{3}=(z t) \partial_{t}+(z x) \partial_{x}+(z y) \partial_{y}+\left(\frac{\left(-x^{2}-y^{2}+z^{2}+t^{2}\right)}{2}+\frac{2}{K}\right) \partial_{x} \\
& X_{4}=\left(\frac{\left(x^{2}+y^{2}+z^{2}+t^{2}\right)}{2}-\frac{2}{K}\right) \partial_{t}+(t x) \partial_{x}+(t y) \partial_{y}+(t z) \partial_{x}
\end{aligned}
$$

and

$$
\begin{aligned}
X_{5} & =x \partial_{t}+t \partial_{x} \\
X_{6} & =y \partial_{t}+t \partial_{y} \\
X_{7} & =z \partial_{t}+t \partial_{z} \\
X_{8} & =y \partial_{x}-x \partial_{y} \\
X_{9} & =z \partial_{x}-x \partial_{z} \\
X_{10} & =z \partial_{y}-y \partial_{z}
\end{aligned}
$$

These vector fields are the Lie symmetry vectors of the geodesic equations:

$$
\frac{d^{2} t}{d s^{2}}+\frac{1}{2} N K t\left[\left(\frac{d t}{d s}\right)^{2}+\left(\frac{d x}{d s}\right)^{2}+\left(\frac{d y}{d s}\right)^{2}+\left(\frac{d z}{d s}\right)^{2}\right]-N K\left(\frac{d t}{d s}\right)\left[x\left(\frac{d x}{d s}\right)+y\left(\frac{d y}{d s}\right)+z\left(\frac{d z}{d s}\right)\right]=0
$$




$$
\begin{aligned}
& \frac{d^{2} x}{d s^{2}}+\frac{1}{2} N K x\left[\left(\frac{d t}{d s}\right)^{2}+\left(\frac{d x}{d s}\right)^{2}+\left(\frac{d y}{d s}\right)^{2}+\left(\frac{d z}{d s}\right)^{2}\right]-N K\left(\frac{d x}{d s}\right)\left[t\left(\frac{d t}{d s}\right)+y\left(\frac{d y}{d s}\right)+z\left(\frac{d z}{d s}\right)\right]=0 \\
& \frac{d^{2} y}{d s^{2}}+\frac{1}{2} N K y\left[\left(\frac{d t}{d s}\right)^{2}+\left(\frac{d x}{d s}\right)^{2}+\left(\frac{d y}{d s}\right)^{2}+\left(\frac{d z}{d s}\right)^{2}\right]-N K\left(\frac{d t}{d s}\right)\left[t\left(\frac{d t}{d s}\right)+x\left(\frac{d x}{d s}\right)+z\left(\frac{d z}{d s}\right)\right]=0 \\
& \frac{d^{2} z}{d s^{2}}+\frac{1}{2} N K z\left[\left(\frac{d t}{d s}\right)^{2}+\left(\frac{d x}{d s}\right)^{2}+\left(\frac{d y}{d s}\right)^{2}+\left(\frac{d z}{d s}\right)^{2}\right]-N K\left(\frac{d z}{d s}\right)\left[t\left(\frac{d t}{d s}\right)+x\left(\frac{d x}{d s}\right)+y\left(\frac{d y}{d s}\right)\right]=0
\end{aligned}
$$

To compare these results with those obtained in [6] we consider the change of coordinates:

$$
\begin{aligned}
& t=2 \tanh \frac{\psi}{2} \cosh r \\
& x=2 \tanh \frac{\psi}{2} \sinh r \cos \theta \\
& y=2 \tanh \frac{\psi}{2} \sinh r \sin \theta \cos \phi \\
& z=2 \tanh \frac{\psi}{2} \sinh r \sin \theta \sin \phi .
\end{aligned}
$$

In the coordinates $(\psi, r, \phi, \theta)$ and assuming $K=1$ the metric reads:

$$
d s^{2}=-d \psi^{2}+\sinh ^{2} \psi\left(d r^{2}+\sinh ^{2} r\left[d \theta^{2}+\sin ^{2} \theta d \phi^{2}\right]\right) .
$$

The geodesic equations become:

$$
\begin{gathered}
\frac{d^{2} \psi}{d s^{2}}+\sinh \psi \cosh \psi\left(\frac{d r}{d s}\right)^{2}+\sinh \psi \cosh \psi \sinh ^{2} r\left(\frac{d \theta}{d s}\right)^{2}+\sinh \psi \cosh \psi \sinh ^{2} r \sin ^{2} \theta\left(\frac{d \phi}{d s}\right)^{2}=0 \\
\frac{d^{2} r}{d s^{2}}+2 \operatorname{coth} \psi\left(\frac{d \psi}{d s}\right)\left(\frac{d r}{d s}\right)-\sinh r \cosh r\left(\frac{d \theta}{d t}\right)^{2}-\sinh r \cosh r \sin ^{2} \theta\left(\frac{d \phi}{d t}\right)^{2}=0 \\
\frac{d^{2} \theta}{d s^{2}}+2 \operatorname{coth} \psi\left(\frac{d \psi}{d s}\right)\left(\frac{d \theta}{d s}\right)+2 \operatorname{coth} r\left(\frac{d r}{d s}\right)\left(\frac{d \theta}{d s}\right)-\sin \theta \cos \theta\left(\frac{d \phi}{d s}\right)^{2}=0 \\
\frac{d^{2} \phi}{d s^{2}}+2 \operatorname{coth} \psi\left(\frac{d \psi}{d s}\right)\left(\frac{d \phi}{d s}\right)+2 \operatorname{coth} r\left(\frac{d r}{d s}\right)\left(\frac{d \phi}{d s}\right)+2 \cot \theta\left(\frac{d \theta}{d s}\right)\left(\frac{d \phi}{d s}\right)=0 .
\end{gathered}
$$

The Lie symmetry vectors of these equations are:

$$
\begin{aligned}
& X_{1}=\left[2 \cos \theta \sinh r,-2 \operatorname{coth} \psi \cos \theta \cosh r, 2 \frac{\operatorname{coth} \psi \sin \theta}{\sinh r}, 0\right] \\
& X_{2}=\left[-2 \sinh r \sin \theta \cos \phi, 2 \operatorname{coth} \psi \cosh r \cos \theta \cos \phi, 2 \frac{\operatorname{coth} \psi \cos \theta \cos \phi}{\sinh r},-2 \frac{\operatorname{coth} \psi \sin \phi}{\sinh r \sin \theta}\right] \\
& X_{3}=\left[-2 \sinh r \sin \theta \sin \phi, 2 \operatorname{coth} \psi \cosh r \sin \theta \sin \phi, 2 \frac{\operatorname{coth} \psi \cos \theta \sin \phi}{\sinh r}, 2 \frac{\operatorname{coth} \psi \cos \phi}{\sinh r \sin \theta}\right] \\
& X_{4}=[-2 \cosh r, 2 \operatorname{coth} \psi \sinh r]
\end{aligned}
$$

and

$$
\begin{aligned}
X_{5} & =[0, \cos \theta,-\operatorname{coth} r \sin \theta, 0] \\
X_{6} & =\left[0, \cos \phi \sin \theta, \operatorname{coth} r \cos \theta \cos \phi,-\frac{\operatorname{coth} r \sin \phi}{\sin \theta}\right] \\
X_{7} & =\left[0, \sin \phi \sin \theta, \operatorname{coth} r \cos \theta \sin \phi, \frac{\operatorname{coth} r \cos \phi}{\sin \theta}\right] \\
X_{8} & =[0,0,-\cos \phi, \cot \theta \sin \phi] \\
X_{9} & =[0,0,-\sin \phi,-\cot \theta \cos \phi] \\
X_{10} & =[0,0,0,-1]
\end{aligned}
$$




\subsection{The geodesic symmetries of Gödel spacetime}

The Gödel metric in Cartesian coordinates is:

$$
d s^{2}=-d t^{2}-2 e^{a x} d t d y+d x^{2}-\frac{1}{2} e^{2 a x} d y^{2}+d z^{2}
$$

The geodesic equations are:

$$
\begin{aligned}
\frac{d^{2} t}{d s^{2}}+2 a \frac{d t}{d s} \frac{d x}{d s}+a e^{a x} \frac{d x}{d s} \frac{d y}{d s} & =0 \\
\frac{d^{2} x}{d s^{2}}+a e^{a x} \frac{d t}{d s} \frac{d y}{d s}+\frac{1}{2} a e^{2 a x}\left(\frac{d y}{d s}\right)^{2} & =0 \\
\frac{d^{2} y}{d s^{2}}-2 a e^{-a x} \frac{d t}{d s} \frac{d x}{d s} & =0 \\
\frac{d^{2} z}{d s^{2}} & =0 .
\end{aligned}
$$

The special projective algebra of the Gödel metric has as follows:

a. KVs

One gradient $\mathrm{KV}$ :

$$
Y^{1}=\partial_{z}, \text { gradient function } z
$$

and four non-gradient KVs:

$$
\begin{aligned}
Y^{2} & =\left(-\frac{2}{a} e^{-a x}\right) \partial_{t}+y \partial_{x}+\left(\frac{2 e^{-2 a r}-a^{2} y^{2}}{2 a}\right) \partial_{y} \\
Y^{3} & =\partial_{x}-a y \partial_{y} \\
Y^{4} & =\partial_{t} \\
Y^{5} & =\partial_{y} .
\end{aligned}
$$

b. No HKV

c. One proper AC [16]:

$$
Y^{6}=z \partial_{z}
$$

d. No special PC [16].

The generic Lie Symmetry vector of the geodesic equations of the Gödel metric is:

$$
\begin{aligned}
\mathbf{X} & =\left(c_{1}+c_{2} s+c_{3} z\right) \partial_{s}+\left(c_{4}+c_{5}\left(-\frac{2}{a} e^{-a x}\right)\right) \partial_{t}+ \\
& +\left(c_{5} y+c_{6}\right) \partial_{x}+\left(c_{5}\left(\frac{2 e^{-2 a r}-a^{2} y^{2}}{2 a}\right)+c_{6}(-a y)+c_{7}\right) \partial_{y}+\left(c_{8}+c_{9} s+c_{10} z\right) \partial_{z}
\end{aligned}
$$

where "s" is an affine parameter along the geodesics. There result ten Lie symmetry vectors as follows:

$$
\begin{gathered}
X_{1}=\partial_{s}, \quad X_{2}=s \partial_{s}, \quad X_{3}=z \partial_{s}, \quad X_{4}=\partial_{t} \\
X_{5}=\left(-\frac{2}{a} e^{-a x}\right) \partial_{t}+x \partial_{x}+\left(\frac{2 e^{-2 a r}-a^{2} x^{2}}{2 a}\right) \partial_{x} \\
X_{6}=\partial_{x}-a x \partial_{x}, \quad X_{7}=\partial_{y}, \quad X_{8}=\partial_{z}, \quad X_{9}=s \partial_{z}, \quad X_{10}=z \partial_{z} .
\end{gathered}
$$

The non-vanishing Lie brackets of these vectors are:

$$
\begin{array}{llll}
{\left[X_{1}, X_{2}\right]=X_{1} \quad,} & {\left[X_{1}, X_{9}\right]=X_{8}} \\
{\left[X_{2}, X_{3}\right]=-X_{3},} & {\left[X_{2}, X_{9}\right]=X_{9}} \\
{\left[X_{3}, X_{8}\right]=-X_{1} \quad, \quad\left[X_{3}, X_{9}\right]=X_{10}-X_{2} \quad, \quad\left[X_{3}, X_{10}\right]=-X_{3}} \\
{\left[X_{5}, X_{6}\right]=X_{5} \quad, \quad\left[X_{5}, X_{7}\right]=-X_{7}} \\
{\left[X_{6}, X_{7}\right]=a X_{7} \quad, \quad\left[X_{8}, X_{10}\right]=X_{8}, \quad\left[X_{9}, X_{10}\right]=X_{9}}
\end{array}
$$


There are two Lie subalgebras. One spanned by the vectors $\left\{X_{1}, X_{4}, X_{5}, X_{6}, X_{7}, X_{8}, X_{9}\right\}$ with non-vanishing commutators:

$$
\begin{aligned}
& {\left[X_{1}, X_{9}\right]=X_{8}, \quad\left[X_{3}, X_{8}\right]=-X_{1} \quad, \quad\left[X_{3}, X_{10}\right]=-X_{3}} \\
& {\left[X_{5}, X_{6}\right]=X_{5} \quad, \quad\left[X_{5}, X_{7}\right]=-X_{7} \quad, \quad\left[X_{6}, X_{7}\right]=a X_{7}}
\end{aligned}
$$

and a second spanned by the vectors $X_{2}, X_{3}, X_{10}$ with non-vanishing commutators.

$$
\left[X_{2}, X_{3}\right]=-X_{3} \quad, \quad\left[X_{3}, X_{10}\right]=-X_{3}
$$

It can be shown that the first subalgebra consists of the Noether symmetries of the Lagrangian $L=$ $\frac{1}{2} g_{i j} \frac{d x^{i}}{d s} \frac{d x^{j}}{d s}$ which gives the geodesic equations. The Noether constant corresponding to the Noether symmetry $X_{1}=\partial_{s}$ is the total energy i.e. the Hamiltonian.

\section{Conclusions}

We derived the symmetry conditions for the admittance of a Lie symmetry by the equations of autoparallels (paths) in an affine space. The important conclusion is that the Lie symmetry vector is a PC in the jet space $\left\{t, x^{i}\right\}$ while the vectors $\eta^{i}(t, x) \partial_{x^{i}}$ are projective collineations in the space $\left\{x^{i}\right\}$. The symmetry conditions are applied to the geodesics of a Riemannian space were they are solved and the generic Lie symmetry vector is obtained in terms of the special projective algebra (and its degeneracies KVs, HKV, ACs) of the metric. It would be useful to to apply these results to the problem of geometric linearization of ODEs [15]. In the current paper we apply the results to Einstein spaces and obtaine the Lie symmetry vectors in terms of the KVs of the metric, in agreement with the conjecture made in [6]. Finally we considered specific examples and computed the Lie symmetries of the De Sitter spacetime and the Gödel metric. 


\section{References}

[1] Hussain I, Mohamed F, Qadir A 2009, Approximate Noether symmetries of the geodesic equations for the charged Kerr- spacetime and rescaling of energy Gen Reltiv Grav 41, 2399 -2414

[2] Aminova, A. V. 1994, "Automorphisms of geometric structures as symmetries of diferential equations" (in Russian), Izv. - Vyssh. - Uchebn. - Zaved. - Mat. 2, 3 - 10

[3] Aminova, A. V. 1995, "Projective transformations and symmetries of differential equations", Sbornik Mathematics 186 (12), 1711 - 1726

[4] Aminova, A. V. 2000, "Projective geometry of systems of differential equations: General conceptions", Tensor, N.S., $65-86$

[5] Aminova, A. V. 1978, Concircular vector fields and group symmetries in spaces of constant curvature (in Russian), Gravit.i. Teoriya Otmotisel'n 14, 4 - 16

[6] Feroze, T., Mahomed, F.M., Qadir, A. 2006, "The connection between isometries and symetries of geodesic equations of the underlying spaces", Nonlinear Dynamics 65 - 74

[7] Prince G E and Crampin M (1984) Gen Rel Grav. 16, 921

[8] We define the sign of the curvature tensor form the identity $A_{; b c}^{a}-A_{; c b}^{a}=R_{b c d}^{a} A^{d}$ or $A_{a ; b c}-A_{a ; c b}=R_{d a b c} A^{d}$. In terms of the connection coefficients $R_{b c d}^{a}=\Gamma_{d b}^{a}, c-\Gamma_{c b}^{a}, d+\Gamma_{c f}^{a} \Gamma_{d b}^{f}-\Gamma_{d f}^{a} \Gamma_{c b}^{f}$.

[9] Yano K 1956 The theory of Lie derivatives and its Applications, (Amsterdam: North Holland)

[10] Stephani, H. 1989 , Differential Equations: Their Solutions using Symmetry, Cambridge University Press, NY

[11] Scouten KJ A 1954 Ricci Calculus, (Berlin: Springer)

[12] Tsamparlis, M., Nikolopoulos, D., Apostolopoulos, P. S. 1998, Class. Quantum Grav., 15, 2909

[13] Barnes, A. 1993, "Projective collineations in Einstein spaces" Class. Quantum Grav., 10, 1139 - 1145

[14] Qadir A. 2007, Geometric linearization of ordinary differential equations, Symmetry, Integrability and Geometry: Methods and Applications (SIGMA) 3, 103 - 109

[15] Mahomed F.M., Qadir A. 2009, Linearization criteria for systems of cubically semi-linear second order differential equations, J. Nonlinear Mathematical Physics 16 , 1 - 16

[16] Hall G.S., da Costa J.(1988), Affine collineations in space-time J. Math. Phys. 29, 2465 - 2472 